\title{
MAPPING CYLINDER NEIGHBORHOODS ${ }^{1}$ )
}

\author{
BY \\ VICTOR NICHOLSON
}

1. Let $X$ be a triangulated 3-manifold and $C$ a subcomplex of $X$. A regular neighborhood of $C$ in $X$ is the union of all simplexes in a second derived subdivision of $X$ that intersect $C$. Every subcomplex $C$ of $X$ has a regular neighborhood. We consider the converse using a generalization of regular neighborhoods.

Let $C$ be a closed subset of a space $X$. A subspace $U$ of $X$ is called a mapping cylinder neighborhood (MCN) of $C$ if $U=f(M \times I) \cup C$ where $f$ is a map of a space $M \times I$ into $X$ such that $f \mid M \times[0,1)$ is a homeomorphism into $X-C, f(M \times 1)$ $=C \cap \mathrm{Cl}(X-C)$ and $f(M \times(0,1]) \cup C$ is open in $X$. As noted in [12], regular neighborhoods are MCN's.

Suppose $C$ is a closed subset of a 3-manifold $X$ and $U=f(M \times I) \cup C$ a MCN of $C$. We note some properties of $U$.

(a) Since $M \times(0,1)$ is a 3-manifold, $M$ is a generalized 2-manifold [17] and thus a 2-manifold [19]. Hence $U$ is a 3-manifold with boundary.

(b) If $C$ is compact then $U$ is compact (Lemma 1).

(c) If $C$ is compact and $U^{\prime}$ is another MCN of $C$ then Int $U$ and Int $U^{\prime}$ are homeomorphic [12]. Thus $U$ and $U^{\prime}$ are homeomorphic [9, Theorem 3].

Our converse: Suppose $X$ is a 3-manifold and $C \subset X$ is a topological complex, i.e., $C$ is homeomorphic to a locally finite simplicial complex. Suppose also that $C$ is closed in $X$ and $C$ has a MCN. Then $C$ must be a subcomplex of some triangulation of $X$.

THEOREM 1. If $C$ is a topological complex which is a closed subset of a 3-manifold $X$, then $C$ is tame if and only if $C$ has a MCN.

Our motivation for Theorem 1 was the special case where $C$ is a 1,2 or 3-cell and $M$ is a 2-sphere [6], [10]. An immediate corollary to Theorem 1 is

THEOREM 2. Suppose $C$ is a tame topological complex in a 3-manifold $X, g$ is a map of $X$ into a 3-manifold $Y$ such that $g^{-1} g(C)=C, g$ is a homeomorphism on $X-C$, and $g(C)$ is a topological complex. Then $g(C)$ is tamely embedded in $Y$.

Proof. By Theorem 1, $C$ has a MCN $U$. The conditions on $g$ guarantee that $g(U)$ is a $\mathrm{MCN}$ of $g(C)$.

A special case of Theorem 1 in dimension four is also immediate. Suppose $N$ is a space, $f: N \rightarrow N$ an onto map, and $N_{f}$ the mapping cylinder defined by $N$ and

Presented to the Society, January 24, 1969; received by the editors November 5, 1968.

( $\left.{ }^{1}\right)$ This paper is part of the author's Ph.D. thesis which was prepared under the supervision of T. M. Price at the University of Iowa. 
$f$. Let $g: N \times I \rightarrow N_{f}$ be the natural map. If there exists a pseudo-isotopy $k_{t}(N)$ $\rightarrow N$ such that $k_{0}=\mathrm{id}$ and $k_{1}=f$, then the map $h: N_{f} \rightarrow N \times I$ defined by $h[g(x, t)]$ $=\left(k_{t}(x), t\right)$ is a homeomorphism. If $G$ is a cellular upper semicontinuous decomposition of a 3-manifold $M$ and $M / G$ is a 3-manifold, then there exists a pseudoisotopy of $M$ onto itself that shrinks the nondegenerate elements to points [18]. Thus such a pseudo-isotopy exists when $N$ and $f(N)$ are 3-manifolds and $f$ is the projection map of a cellular upper semicontinuous decomposition of $N$. We have

THEOREM 3. Suppose $N$ is a compact connected 3-manifold in a 4-manifold $Y$. Suppose $M$ is a 3-manifold and $U=f(M \times I)$ is a MCN of $N$ where the restriction of $f$ to each component of $M \times 1$ is a cellular map. Then $U$ is a bicollar for $N$ in $Y$.

Is the cellularity condition given in Theorem 3 implied by the fact that $Y$ is a 4-manifold? This is the case in $\operatorname{dim} 3$; see Lemma 3(2).

2. Proof of Theorem 1. We have placed the lemmas in the sections following the proof.

Proof. Suppose $C$ has a MCN. By the procedure described at the first of the proof of Lemma 6 the union of the 1 and 2-skeleton of $C$ has a MCN. Thus the interior of each 2-simplex is tame by Lemma 3(3). By Lemmas 5 and 6 the 1skeleton of $C$ is tame. In particular, the boundary of each 2-simplex in $C$ is tame. It is a consequence of Lemmas 5.1 and 5.2 of [13] that a disk is tame if its interior and boundary are tame. Therefore the star of each vertex in $C$ is tame, since each 2-simplex is tame and the 1-skeleton is tame [7, Theorem 3.3]. Thus $C$ is locally tame and hence tame [3]. Suppose $C$ is tame. Then $C$ has a regular neighborhood under some triangulation of $X$. This regular neighborhood is a MCN. This completes the proof.

2.a. Suppose $C$ is a closed subset of a 3-manifold $X$ and $U=f(M \times I) \cup C$ is a MCN of $C$. We let $F=f \mid M \times 1$ and $i_{t}(t \in I)$ denote the identification of $M \times t$ with $M$. For example, if $x \in C \cap \mathrm{Cl}(X-C)$ then $f\left(i_{1} F^{-1}(x) \times 0\right) \subset \mathrm{Bd} U$.

Lemma 1. Suppose $C$ is a closed subset of a 3-manifold $X$ and $U=f(M \times I) \cup C$ is a MCN of $C$. (1) If $A$ is open in $F(M \times 1)$ and contractible then every simple closed curve in $F^{-1}(A)$ separates $F^{-1}(A)$. (2) If $A \subset C$ is compact then $F^{-1}(A)$ is compact.

Proof. Suppose not. There exist simple closed curves $S_{1}$ and $S_{2}$ in $f\left(i_{1} F^{-1}(A) \times 0\right)$ $\subset \mathrm{Bd} U$ which intersect in one point and cross there. Both curves are inessential in $U$ because of the mapping cylinder structure over $A$. A simple closed curve which bounds a singular disk has a neighborhood homeomorphic to a solid torus. Thus $S_{1} \cup S_{2}$ has an orientable neighborhood in Bd $U$. This is a contradiction. A 3-manifold with boundary having orientable boundary cannot contain two inessential simple closed curves in its boundary which cross at an odd number of points. See [11, p. 29] or [15, Lemma 6.1].

(2) The local compactness of the MCN implies that $F$ is a compact map. For let $T$ be any compact subset of $C$. There exists an open set $Q \subset U$ such that $T \subset Q$ 
$\subset \bar{Q} \subset$ Int $U$ and $\bar{Q}$ is compact. For each point $p \in F^{-1}(T)$, the arc $f\left(i_{1}(p) \times I\right)$ intersects $\bar{Q}-Q$. Since $F^{-1}(T)$ is closed, $J=f\left(i_{1} F^{-1}(T) \times I\right) \cap(\bar{Q}-Q)$ is compact. Thus $F^{-1}(T)$ is compact, since the projection of $M \times I$ onto $M \times 1$ carries $f^{-1}(J)$ onto $F^{-1}(T)$.

2.b. 2-simplexes. First some definitions. Let $x \in L$ where $L$ is a 2-manifold with boundary in a 3-manifold $X$. The local separation theorem [1, $\$ 2$, Corollary 2] yields: For every $\varepsilon>0$, there exists an $\varepsilon$-neighborhood $N$ of $x$ in $X$ such that $N-L$ has two components $O_{1}$ and $O_{2}$. If $x \in \mathrm{Bd} L$, then $O_{2}=\varnothing$. If $x \in \operatorname{Int} L$, then $O_{1}$ and $\mathrm{O}_{2}$ are nonempty. We say $U^{\prime} \subset X$ is a 1-sided neighborhood of $x$ if there exists a neighborhood $N$ of $x$ from the local separation theorem such that $O_{1} \cup(N \cap L)$ $\subset U^{\prime}$ and $O_{2} \cap U^{\prime}=\varnothing$.

Let $C$ be a topological complex which is closed in $X$ and consists of 1 and 2simplexes. Let $U=f(M \times I)$ be a MCN of $C$ and $\Delta$ a 2-simplex of $C$. We say $U$ contains a 1-sided $M C N, U^{\prime}$, of $x \in$ Int $\Delta$ if there exists a disk $D \subset M$ such that $U^{\prime}=f(D \times I)$ is a 1-sided neighborhood of $X$. We shall show in Lemma 3 that 1-sided MCN's always exist. Lemma 2 is a standard type of result for 2-manifolds; we omit a proof.

LEMMA 2. Let $M$ be a 2-manifold, $B$ a nonempty, proper open connected subset of $M$ such that $\bar{B}$ is compact and every simple closed curve in $B$ separates $B$. If $E$ is a continuum in $B$ and $K$ is a continuum in $B$ which separates $E$ from $\mathrm{Bd} B$, then $E$ lies in the interior of a disk $D \subset B$.

Consider a fixed $x \in \operatorname{Int} \Delta$. We distinguish two sets, $H$ and $L$, in $M \times 1$ which correspond to the two sides of $\Delta$ near $x$. Let $N, O_{1}$ and $O_{2}$ be given for $x$ by the local separation theorem. Let $P$ be a disk such that $x \in \operatorname{Int} P \subset P \subset(N \cap$ Int $\Delta)$ and $z \in \operatorname{Int} P$. For $y \in F^{-1}(z)$, let $A_{y}$ denote the $\operatorname{arc} f\left(i_{1}(y) \times I\right)$. There exists a first point $p$ from $z$ in $A_{y} \cap(\bar{N}-N)$. Then $[z, p) \subset N$ and $(z, p) \subset O_{1}$ or $O_{2}$. We say $A_{y}$ ends through $O_{1}$ or $O_{2}$, respectively. Let $H_{z}\left(L_{z}\right)$ be the set of all points $y$ such that $A_{y}$ ends through $O_{1}\left(O_{2}\right)$. Let $H=\bigcup H_{z}, L=\bigcup L_{z}, z \in \operatorname{Int} P$.

We show $H$ and $L$ are open and separated. We assume the neighborhood $N$ was chosen to lie inside a neighborhood $Q$ of $x$ homeomorphic to $E^{3}$. Suppose there exist $y \in H$ and $b \in L$ lying in the same component of $F^{-1}($ Int $P$ ). There exist an arc $b y \subset F^{-1}($ Int $P$ ) and an $\operatorname{arc} F(b) F(y) \subset$ Int $P$. There exists $0<t<1$ so that the arc $f\left(i_{1}(b y) \times t\right)$ together with $F(b) F(y)$ and subarcs of $A_{y}$ and $A_{b}$ form a simple closed curve $S \subset Q$. Since $A_{y}$ ends through $O_{1}$ and $A_{b}$ ends through $O_{2}, S$ links Bd $P$ (homology linking mod 2; see [4]). But since $b y \subset F^{-1}($ Int $P$ ), $S$ can be shrunk to a point in $Q-\mathrm{Bd} P$ by first pulling it into Int $P$ using the mapping cylinder. Contradiction. Therefore $H$ and $L$ are the union of components of $F^{-1}(\operatorname{Int} P)$. Thus they are open and separated.

Lemma 3. Suppose $C$ is a topological complex which is a closed subset of a 3manifold $X$. Suppose $C$ consists of 1 and 2-simplexes and $U=f(M \times I)$ is a $M C N$ of $C$. Also suppose $\Delta$ is a 2-simplex in $C$ and $x \in \operatorname{Int} \Delta$. Then (1) $U$ contains a 1-sided MCN 
of $x$ on each side of $\Delta$ and the two disks defining the MCN's are disjoint, (2) $H_{x}$ and $L_{x}$ are cellular in $M$, and (3) Int $\Delta$ is locally tame.

Proof. (1) Let $x$ be a distinguished point in Int $\Delta$ and $N, O_{1}$ and $O_{2}$ be given for $x$ by the local separation theorem such that $N$ lies in a neighborhood of $x$ homeomorphic to $E^{3}$. Let $P$ be a disk such that $x \in \operatorname{Int} P \subset P \subset(N \cap$ Int $\Delta)$ and let $H$ and $L$ be given as in the discussion preceding the lemma. We consider only $H$. By Lemma $1, F^{-1}(x)$, and hence $H_{x}$, is compact. If $H_{x}$ were not connected we could separate two of its components, say $T_{1}$ and $T_{2}$, in $M \times 1$ with a finite number of simple closed curves $S_{i} \subset H$. But points in $f\left(T_{1} \times I\right)$ and $f\left(T_{2} \times I\right)$ can be joined by small arcs in $O_{1}$. A contradiction is reached since $f\left(\bigcup S_{i} \times[0,1)\right)$ separates $f(M \times[0,1))$ and $x$ is not a limit point of $f\left(\cup S_{i} \times I\right)$. Thus $F \mid H$ is monotone. There exist disks $D_{1}$ and $D_{2}$ such that $x \in$ Int $D_{1} \subset D_{1} \subset$ Int $D_{2} \subset D_{2} \subset$ Int $P$. Let $E=H_{x}$ and $B=\bigcup H_{y}, y \in \operatorname{Int} D_{2}$. Let $K=\bigcup H_{y}, y \in \mathrm{Bd} D_{1}$. The map $F$ is closed on $F^{-1}\left(D_{2}\right) \cap H$. The inverse image of a connected set is connected under a monotone closed map. Thus the sets $E, K$ and $B$ satisfy the hypothesis of Lemma 2. Let $D \subset M \times 1$ be a disk given by Lemma 2. Then $U^{\prime}=f\left(i_{1}(D) \times I\right)$ is a 1 -sided MCN of $x$. For since $D \subset H$, there exists $t<1$ such that $f\left(i_{1}(D) \times[t, 1]\right) \subset O_{1}$. Picking a neighborhood $N(q)$ of $x$ by the local separation theorem such that $N(q) \subset N$ and $N(q) \cap f\left(i_{1}(D) \times[0, t]\right)=\varnothing$, we have $O_{2}(q) \cap f\left(i_{1}(D) \times I\right)=\varnothing$. Since $H_{x} \cap \mathrm{Bd} D=\varnothing$ and $i_{1}(\mathrm{Bd} D) \times I$ separates $M \times I$, there exists a neighborhood $N(r)$ of $x$ from the local separation theorem such that $O_{1}(r) \subset U^{\prime}$. For a neighborhood $N(s)$ of $x$ from the local separation theorem contained in $N(q) \cap N(r)$ we have $O_{1}(s) \subset U^{\prime}$ and $O_{2}(s) \cap U^{\prime}=\varnothing$. Therefore $U^{\prime}$ is a 1-sided MCN of $x$. A similar argument using $L$ yields a disk disjoint from $D$ and a 1-sided MCN of $x$ on the $\mathrm{O}_{2}$ side of $\Delta$.

(2) Let $N(s)$ be the neighborhood of $x$ given above and $D_{3}$ a disk such that $x \in$ Int $D_{3} \subset D_{3} \subset(N(s) \cap$ Int $\Delta)$. Then $H \cap F^{-1}\left(\right.$ Int $\left.D_{3}\right)$ is an open connected subset of Int $D$ and not separated by $H_{x}$. Thus $H_{x}$, and similarly $L_{x}$, is cellular.

(3) We shall show that $X$-Int $\Delta$ is locally simply connected at $x$. Consider the 1 -sided MCN of $x, U^{\prime}=f\left(i_{1}(D) \times I\right)$ and let $\varepsilon>0$. There exists an $\varepsilon$-neighborhood $N(\varepsilon)$ of $x$ from the local separation theorem such that $O_{1}(\varepsilon) \subset U^{\prime}$ and $O_{2}(\varepsilon) \cap U^{\prime}$ $=\varnothing$. Since $F^{-1}\left(N(\varepsilon) \cap U^{\prime}\right)$ is open in $i_{1}(D) \times I$ and $H_{x}$ is cellular, there exists a disk $G \subset i_{1}(D)$ and a number $t<1$ such that $G \times[t, 1] \subset F^{-1}\left(N(\varepsilon) \cap U^{\prime}\right)$ and $H_{x} \subset$ Int $G \times 1$. Let $T=(\operatorname{Int} G) \times(t, 1]$. There exists a neighborhood $Q$ of $x$ from the local separation theorem such that $Q \subset N(\varepsilon)$ and $Q \cap f((D \times I)-T)=\varnothing$. The component $O_{1}(q)$ of $Q$-Int $\Delta$ lies in $f(T)$. Let $J$ be any simple closed curve in $O_{1}(q)$. There exists $r<1$ such that $f(D \times r)$ separates $J$ from $F(D)$ in $f\left(i_{1}(D) \times I\right)$. Thus $J$ can be shrunk to a point in the interior of the 3-cell $f(G \times[t, r]) \subset N(\varepsilon)$. Using the 1-sided MCN of $x$ on the other side of Int $\Delta$, we have that $X$-Int $\Delta$ is locally simply connected at $x$. Since $X$-Int $\Delta$ is locally simply connected at each $x \in$ Int $\Delta$, Int $\Delta$ is locally tame [5]. 
2.c. 1-complexes. Let $n$ be a positive integer. An $n$-frame $T$ is the union of $n$ arcs $A_{i}=\left[p, a_{i}\right]$ such that $A_{i} \cap A_{j}=p$. The points $a_{i}$ are the endpoints of $T$. The interior of $T$, Int $T$, is $T$ minus its endpoints. We define a $M C N$ of the interior of $T$. No confusion should result from this different use of MCN. Let $S^{2}$ denote the 2-sphere and $D_{i}, i=1, \ldots, n$, be disjoint disks in $S^{2}$. Let $M=S^{2}-\cup D_{i}$ and consider $M \times I$ as a subspace of $S^{2} \times I$. If $T$ is an $n$-frame in a 3-manifold $X$ then Int $T$ is said to have a MCN, $U=f(M \times I)$, if there exists a map $f$ of $M \times I$ into $X$ such that (1) $f \mid M \times[0,1)$ is a homeomorphism into $X-T$, (2) $f(M \times 1)=\operatorname{Int} T$, (3) $U$ is a neighborhood of Int $T$ in $X$, and (4) for any sequence $\left\{b_{j}\right\}$ in $M \times I$ which converges to a point of $\mathrm{Bd} D_{i} \times 1,\left\{f\left(b_{j}\right)\right\}$ converges to the endpoint $a_{i}$ of $T$.

Lemma 4. Suppose $T$ is an $n$-frame in a 3-manifold $X$. If there exists a $M C N$, $f(M \times I)$, of Int $T$ then Int $T$ is locally tame.

Proof. The proof of Lemma 4 follows the procedure used to prove Theorem 1 in [6]. We partition a neighborhood of Int $T$ and a neighborhood of the interior of a standard $n$-frame in $E^{3}$ into homeomorphic pieces. We then obtain a homeomorphism between the neighborhoods which carries $T$ onto the standard $n$-frame. Since for each $t \in(0,1), f(M \times t)$ is bicollared, we may assume that $f(M \times 0)$ is locally tame. Let $C$ be a circle, $A=C \times(0,1) \times I$ and $(x, y, z) \in A$ such that $x \in C$, $y \in(0,1)$ and $z \in I$. Let $B$ denote the half-open annulus in $A, B=\{(x, y, z): y$ $=1 / 2 z+1 / 2\}$.

The properties given in Lemma 1 also hold for a MCN of Int $T$. It therefore follows that $F$ is closed and monotone. Thus the inverse image under $F$ of any connected subset of Int $T$ is connected. For each $i, F^{-1}\left(\operatorname{Int} A_{i}\right)$ is a component of $(M \times 1)-F^{-1}(p)$ and $F^{-1}\left(\right.$ Int $\left.A_{i}\right) \cup D_{i}$ is a component of $\left(S^{2} \times 1\right)-F^{-1}(p)$. Since each component of $S^{2}$ minus the continuum $F^{-1}(p)$ is homeomorphic to $E^{2}$, subtracting the disk $D_{i}$ yields that $F^{-1}\left(\operatorname{Int} A_{i}\right)$ is an open annulus. Thus there exist homeomorphisms $k_{1}$ and $k_{2}$ of $A$ into $i_{1} F^{-1}\left(\operatorname{Int} A_{i}\right) \times I$ such that $B\left(a_{i}\right)=\mathrm{Cl}\left(f k_{1}(B)\right)$ is a disk with $B\left(a_{i}\right) \cap T=a_{i}, B\left(p_{i}\right)=\mathrm{Cl}\left(f k_{2}(B)\right)$ is a disk with $B\left(p_{i}\right) \cap T=p$, Int $B\left(a_{i}\right)-a_{i}$ and Int $B\left(p_{i}\right)-p$ are locally tame, and $B\left(p_{i}\right) \cap B\left(a_{i}\right)=\varnothing$. Similarly, for each $x \in \operatorname{Int} A_{i}, F^{-1}\left(\right.$ Int $\left.A_{i}\right)-F^{-1}(x)$ is the union of two disjoint open annuli. By mapping $A$ homeomorphically into one of these annuli we can define a disk $B(x)$ such that $B(x) \cap T=x$ and $B(x)-x$ is locally tame. For distinct $x$ and $y$ in $A_{i}$ there exist numbers $t_{1}<t_{2}<1$ such that if $W=\mathrm{Cl}\left(f\left(M \times\left[t_{1}, 1\right]\right)-f\left(M \times\left[t_{2}, 1\right]\right)\right)$ and if $P$ is the closure of the component of $f(M \times I)-(B(x) \cup B(y))$ that intersects Int $A_{i}$ then $W \cap P$ is a tame solid torus. Let $O$ be the closure of the component of $f(M \times I)-\bigcup B\left(a_{i}\right)$ that contains Int $T$. Let $L_{i}$ be the closure of the component of $O-\cup B\left(p_{i}\right)$ that contains Int $A_{i}$ and $L=\mathrm{Cl}\left(O-\cup L_{i}\right)$. Let $O^{\prime}$ be the unit ball in $E^{3}$ and $T^{\prime}$ an $n$-frame whose vertex is the origin, whose endpoints lie in $\operatorname{Bd} O^{\prime}$ and which is composed of straight line segments. Partition $O^{\prime}$ into regions $L^{\prime}$ and $L_{i}^{\prime}$ corresponding to $L$ and $L_{i}$. It follows from the proof of Theorem 1 of [6] that we may partition $L_{i}-A_{i}$ and $L_{i}^{\prime}-A_{i}^{\prime}$ into tame solid tori as above whose diameters 
go to zero as the tori approach $T$ in such a way that a homeomorphism $R_{i}: L_{i} \rightarrow L_{i}^{\prime}$ can be obtained by defining homeomorphisms on corresponding tori. Let $J_{1}$ $=f(M \times[0,1 / 2]) \cap L, J_{j}=f(M \times[1 / j, 1 / j+1]) \cap L, j \geqq 2$. Each $J_{j}(j \geqq 1)$ has tame boundary and is homeomorphic to $M \times I$. There exists a collection of regions $\left\{J_{j}^{\prime}\right\}$ in $L^{\prime}$ and a sequence of onto homeomorphisms $S_{j}(j \geqq 1)$ such that $S_{j}: J_{j} \rightarrow J_{j}^{\prime}$ and $S_{j}$ extends $R_{i}(i=1, \ldots, n)$ and $S_{k}(k<j)$. The union of the $S_{j}$ and the $R_{i}$ can be extended to a homeomorphism of $O$ onto $O^{\prime}$ that carries $T$ onto $T^{\prime}$. Thus Int $T$ is locally tame.

Lemma 5. Suppose $C$ is a topological 1-complex which is a closed subset of a 3-manifold $X$. Suppose $C$ has a $M C N, f(M \times I)$. Then $C$ is tame.

Proof. Let $p$ be a vertex of $C$ and $T$ the $n$-frame consisting of all simplexes in $C$ containing $p$. We shall show that $f\left(i_{1} F^{-1}(\operatorname{Int} T) \times I\right)$ is a MCN of Int $T$. It follows from Lemma 1 that $F$ is closed and monotone. Let $\left[p, a_{i}\right]$ be a 1 -simplex in $T$. Let $x, y \in\left(p, a_{i}\right)$ and $q \in(x, y)$. Let $K_{z}=f\left(i_{1} F^{-1}(z) \times 0\right), z=x, y$ and $q$. There exist simple closed curves $S$ and $S^{\prime}$ which separate $K_{q}$ from $K_{x}$ and $K_{q}$ from $K_{y}$ in $f\left(i_{1} F^{-1}[x, y] \times 0\right)$, respectively. The curves $S$ and $S^{\prime}$ can be shrunk to points on disjoint subsets of $f(M \times(0,1]) \cup S \cup S^{\prime}$. They therefore bound disjoint disks there by Dehn's Lemma [16]. Let $K$ be the union of the two disks and the component of $f\left(i_{1} F^{-1}[x, y] \times 0\right)-\left(S \cup S^{\prime}\right)$ that contains $K_{q}$. Since a simple closed curve in $K$ can be pushed off of the two disks, we can obtain from Lemma 1 that every simple closed curve in $K$ separates $K$. Thus $K$ is a 2-sphere [2]. It follows that $F^{-1}\left(p, a_{i}\right)$ is an open annulus. Thus for each $i$, there exists a disk $B_{i}$ in $f\left(i_{1} F^{-1}\left(p, a_{i}\right) \times I\right) \cup a_{i}$ constructed as in the proof of Lemma 4 such that $B_{i} \cap T=a_{i}$. The component of $f(M \times 0)-\cup \mathrm{Bd} B_{i}$ which contains $f\left(i_{1} F^{-1}(p) \times 0\right)$ is a sphere with $n$-holes since its union with the disks $B_{i}$ is a 2 -sphere (again by Lemma 1 and [2]). By the construction of the $B_{i}, F^{-1}(\operatorname{Int} T)$ is a sphere with $n$-holes. It follows that $f\left(i_{1} F^{-1}\right.$ (Int) $\left.T \times I\right)$ is a MCN of Int $T$. By Lemma $4, C$ is locally tame and hence tame [3].

Lemma 6. Suppose $C$ is a topological complex which is a closed subset of a 3manifold X. If C has a MCN then the 1-skeleton of C has a MCN.

Proof. Let $M^{\prime}$ be a 2-manifold and $f^{\prime}$ a map of $M^{\prime} \times I$ into $X$ such that $f^{\prime}\left(M^{\prime} \times I\right)$ $\cup C$ is a MCN of $C$. Let $\left\{K_{i}\right\}$ be the collection of all 3-simplexes in $C$. Let $N_{i}$ be a layer in the collar for Bd $K_{i}$ in $K_{i}$. Let $M=M^{\prime} \cup\left\{N_{i}\right\}$ and $f: M \times I \rightarrow X$ be such that $f\left|M^{\prime} \times I=f^{\prime}, f\right| N_{i} \times I$ is a homeomorphism onto the region between $N_{i}$ and Bd $K_{i}$, and $f\left(N_{i} \times 0\right)=N_{i}$. Then $f(M \times I) \cup C$ is a MCN of $C_{0}$, the union of the 1- and 2-skeleton of $C$. Having removed the 3-simplexes we proceed to eliminate the 2-simplexes. Let $\Delta$ be a 2-simplex in $C_{0}$. We shall show there exists a 2-manifold $M_{1}$ and a map $H_{1}$ of $M_{1} \times I$ into $X$ such that $H_{1}\left(M_{1} \times I\right)$ is a MCN of $C_{0}$-Int $\Delta$ and $H_{1}\left(M_{1} \times I\right)$ agrees with $f(M \times I)$ outside of $f\left(i_{1} F^{-1}(\right.$ Int $\left.\Delta) \times I\right)$. Defining such a map for each 2-simplex in $C_{0}$ will yield a $\mathrm{MCN}$ of the 1-skeleton of $C$. Let 
$p \in$ Int $\Delta$. By Lemma 3 there exist disjoint disks $D_{i}(i=1,2)$ in $M$ such that $F\left(D_{i} \times 1\right) \subset$ Int $\Delta$ and $U_{i}=f\left(D_{i} \times I\right)$ are 1-sided MCN's of $p$ on opposite sides of $\Delta$. Let $\Delta_{1}$ and $\Delta_{2}$ be disks lying in the intersection of the interiors of $F\left(D_{1} \times 1\right)$ and $F\left(D_{2} \times 1\right)$ such that $p \in \operatorname{Int} \Delta_{1} \subset \Delta_{1} \subset$ Int $\Delta_{2}$.

The $M C N$ of $C_{0}$-Int $\Delta_{2}$. Intuitively, we bore a hole through the MCN. Let $A=F^{-1}\left(\mathrm{Bd} \Delta_{1}\right) \cap\left(D_{1} \times 1\right)$ and $J=F^{-1}\left(\mathrm{Bd} \Delta_{2}\right) \cap\left(D_{1} \times 1\right)$. The region between $A$ and $J$ is an open annulus. Let $B$ be a simple closed curve lying in this region and concentric to $A$ and $J$. There exists a map $k$ of $D_{1} \times I$ onto itself that carries each region in Figure 1 onto the corresponding region (labeled with a prime) in Figure $2, k$ is fixed on the boundary of $D_{1} \times I$ and the region labeled $h$ is collapsed into

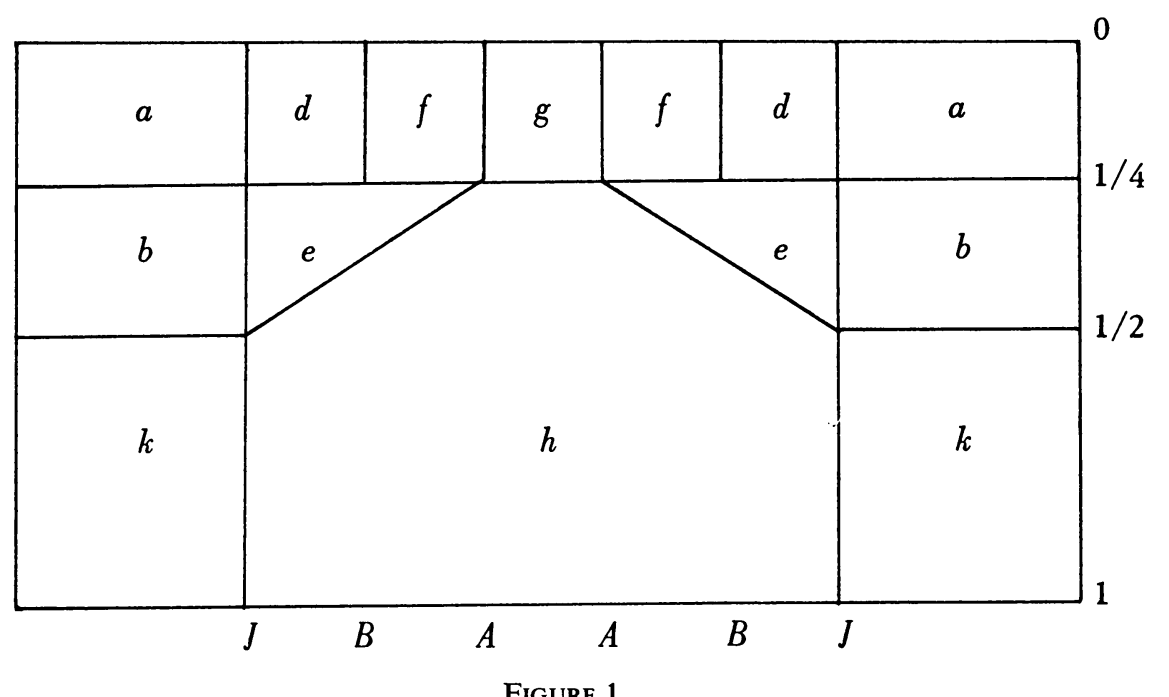

Figure 1

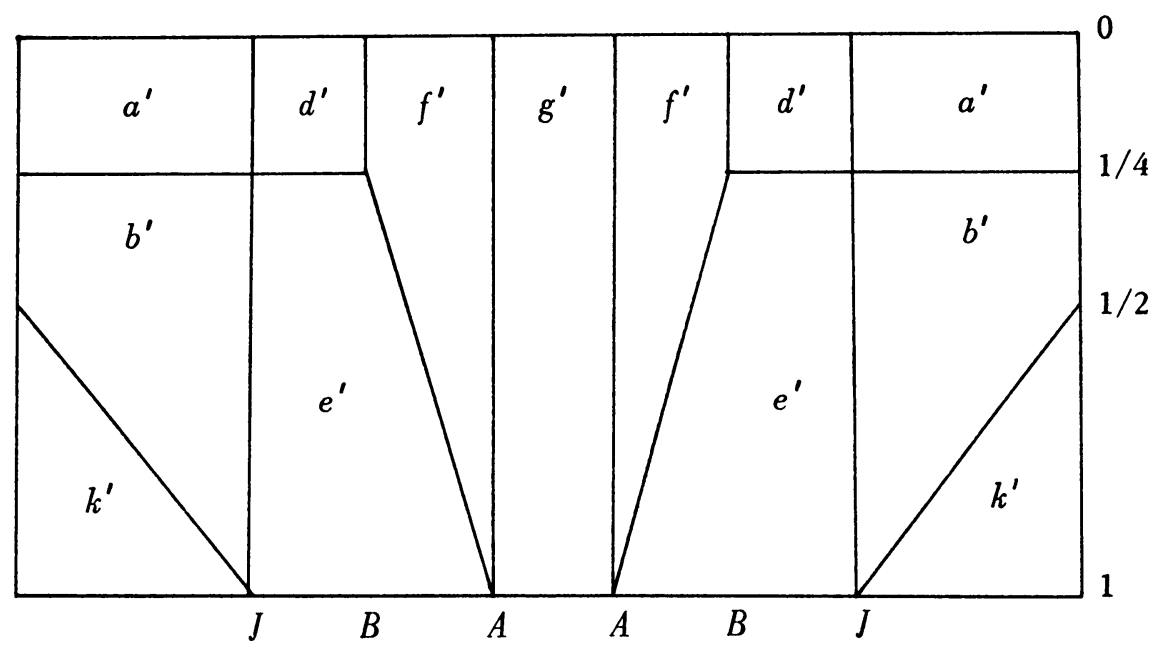

FIGURE 2 
$D_{1} \times 1$. The map $k$ may be extended to map $D_{2} \times I$ onto itself in the same manner as $k$ maps $D_{1} \times I$ onto itself. The spaces $f k\left(\right.$ Int $\left.D_{i} \times t\right), t=1 / 2,1 / 4 ; i=1,2$, are each homeomorphic to $E^{2}$ because they are homeomorphic to spaces of cellular upper semicontinuous decompositions of $E^{2}$, by Lemma 3(2) and [14]. Let $E_{1}$ denote the annulus

$$
f\left\{\left(\left[\mathrm{Bd} D_{1}, i_{1}(B)\right] \times 0\right) \cup\left(i_{1}(B) \times[0,1 / 4]\right) \cup k\left(\left[i_{1}(B), i_{1}(A)\right] \times 1 / 4\right)\right\} \cup \operatorname{Bd} \Delta_{1}
$$

and $T_{1}$ the torus

$$
f\left\{\left(\left(\operatorname{Bd} D_{1}\right) \times[0,1 / 2]\right) \cup k\left(\left[\mathrm{Bd} D_{1}, i_{1}(J)\right] \times 1 / 2\right)\right\} \cup\left[\operatorname{Bd} \Delta_{1}, \mathrm{Bd} \Delta_{2}\right] \cup E_{1} .
$$

It follows from Lemmas 5.1 and 5.2 of [13] that $T_{1}$ is tame. Let $E_{2}$ and $T_{2}$ be the corresponding annulus and torus in $U_{2}$. Let $M_{1}$ be $f(M \times 0)$ minus $f\left(\right.$ Int $\left.D_{1} \times 0\right)$ $\cup f\left(\right.$ Int $\left.D_{2} \times 0\right)$ plus $E_{1} \cup E_{2}$. A homeomorphism $\beta$ may be defined to map $\left(E_{1} \cup E_{2}\right) \times I$ onto the tori $T_{1}$ and $T_{2}$ plus their interiors such that the extension of $\beta$ on $M_{1} \times I$ agrees with $f$ and yields a $\mathrm{MCN}$ of $C_{0}-\operatorname{Int} \Delta_{2}$.

The $M C N$ of $C_{0}$ - Int $\Delta$. We show there exists a map $P$ of $X$ onto itself which collapses the annulus [ $\mathrm{Bd} \Delta_{2}, \mathrm{Bd} \Delta$ ] onto $\mathrm{Bd} \Delta, P$ is a homeomorphism on $X-\left[\operatorname{Bd} \Delta_{2}, \operatorname{Bd} \Delta\right)$, and $P$ moves no point of $X-f\left(F^{-1}(\right.$ Int $\left.\Delta) \times I\right)$. Letting $H_{1}=P \beta$ will give us that $H_{1}\left(M_{1} \times I\right)$ is a $\mathrm{MCN}$ of $C_{0}$-Int $\Delta$. The following spaces are described in cylindrical coordinates in $E^{3}$. Let $S$ be the simple closed curve $(r=1 / 4$, $z=0)$. Let $L$ be the solid annulus $(1 / 4 \leqq r \leqq 1,-1 \leqq z \leqq 1)$. Let $P^{\prime}$ be the map of $L$ onto itself defined by

$$
\begin{aligned}
P^{\prime}(r, \theta, z) & =(r+r(1-|z|), \theta, z), & & 0 \leqq r \leqq 1 / 2, \\
& =(r+(1-r)(1-|z|), \theta, z), & & 1 / 2 \leqq r \leqq 1 .
\end{aligned}
$$

The map $P^{\prime}$ is a homeomorphism on $\mathrm{Bd} L$ and collapses the annulus $(1 / 2 \leqq r \leqq 1$, $z=0)$ into the simple closed curve $(r=1, z=0)$. Let $S_{1}$ be a simple closed curve lying in the annulus $\left[\mathrm{Bd} \Delta_{1}, \mathrm{Bd} \Delta_{2}\right.$ ] concentric to $\mathrm{Bd} \Delta_{1}$. There exists a homeomorphism $\alpha$ of the annulus $(1 / 4 \leqq r \leqq 1, z=0)$ onto the annulus $\left[S_{1}, \operatorname{Bd} \Delta\right]$ in $\Delta$ such that $\alpha(1 / 4, \theta, 0) \in S_{1}, \alpha(1 / 2, \theta, 0) \in \mathrm{Bd} \Delta_{2}$ and $\alpha(1, \theta, 0) \in \mathrm{Bd} \Delta$, for every $\theta$. Since Int $\Delta$ is locally tame there exists a homeomorphism $g$ of $L$ into $X$ such that $g$ extends $\alpha$ and $(g(L)-\mathrm{Bd} \Delta) \subset \beta\left(M_{1} \times(0,1]\right) \cap f\left(i_{1} F^{-1}\right.$ (Int $\left.\left.\Delta\right) \times I\right)$. The required map $P$ is: $P(w)=g P^{\prime} g^{-1}(w)$ for $w \in g(L)$ and the identity elsewhere. This completes the proof.

3. 1-sided MCN's. Let $L$ be a 2-manifold with boundary in a 3-manifold $X$. Let $x \in L, U$ a 1-sided neighborhood of $x$ and $N, O_{1}$ and $O_{2}$ given for $U$. We say $X-L$ is locally simply connected on the $U$ side of $L$ at $x$ if for every $\varepsilon>0$, there exists a neighborhood $N(\varepsilon)$ of $x$ from the local separation theorem such that $N(\varepsilon) \subset N$ and any simple closed curve in $O_{1}(\varepsilon)$ can be shrunk to a point in $X-L$ on a set of diameter less than $\varepsilon$. If $x \in \operatorname{Int} L(x \in \operatorname{Bd} L)$ then $L$ is said to be locally tame from the $U$ side at $x$ if $x$ has a neighborhood in $U$ homeomorphic to a 3-cell 
( $C$ is locally tame at $x$ ). It follows from Theorems 4 and 8 of [5] that $L$ is locally tame from the $U$ side at $x$ if $L$ is locally simply connected on the $U$ side at each point in a neighborhood of $x$. Let $D$ be a disk. A point $x \in L$ is said to have a 1-sided $M C N, U=f(D \times I)$, if there exists a map $f: D \times I \rightarrow X$ such that $f(D \times 1) \subset L$, $f \mid D \times[0,1)$ is a homeomorphism into $X-L$, and $U$ is a 1-sided neighborhood of $x$.

THEOREM 4. Suppose $L$ is a 2-manifold with boundary in a 3-manifold $X$. If $x \in L$ and $x$ has a 1-sided MCN then $L$ is locally tame from the $U$ side at $x$.

Proof. The proof of Lemma 3(3) essentially shows that $X-L$ is locally simply connected on the $U$ side at $x$ for $x \in \operatorname{Int} L$. The case for $x \in \operatorname{Bd} L$ is a consequence of Theorem 1. Consider a small neighborhood of $x$ in $L$ as the topological complex and let $X$ be a properly chosen subset of the 1-sided MCN.

We give a short proof of a result which is part of the folklore of upper semicontinuous decompositions.

THEOREM 5. Suppose $L$ is a 3-manifold with boundary and $G$ an upper semicontinuous decomposition of $L$ all of whose nondegenerate elements lie in $\mathrm{Bd} L$ and are cellular in $\mathrm{Bd} L$. Then $L / G$ is a 3-manifold with boundary.

Proof. If $G$ is an upper semicontinuous decomposition of $E_{+}^{3}$ all of whose nondegenerate elements lie in $E^{2}$ and are cellular in $E^{2}$, then $E^{2} \cdot G$ has a neighborhood in $E_{+}^{3} / G$ homeomorphic to $E_{+}^{3}$. For consider $E_{+}^{3} \subset E^{3}$; then $E^{2} / G$ is homeomorphic to $E^{2}$ by [14] and $E^{3} / G$ is homeomorphic to $E^{3}$ by [8]. Let $P$ denote the projection map of $E^{3}$ onto $E^{3} / G$. For each $x \in E^{2} / G$, there exists a disk $D$ such that $P^{-1}(x)$ $\subset$ Int $D$. Let $U=\{(x, y, z):(x, y, 0) \in D, 0 \leqq z \leqq 1\}$. Then $P(U)$ is a 1 -sided MCN of $x$ in $E_{+}^{3} / G$. By Theorem $4, x$ has a 3-cell neighborhood in $P(U)$. Hence $E_{+}^{3} / G$ contains a neighborhood of $E^{2} / G$ homeomorphic to $E_{+}^{3}$.

Let $h$ be the projection map of $L$ onto $L / G$ and $x \in \mathrm{Bd} L / G$. There exists a neighborhood $Q$ of $h^{-1}(x)$ in $\mathrm{Bd} L$ which is homeomorphic to $E^{2}$ and is the union of elements of $G$. There exists a neighborhood $B$ of $Q$ in $L$ homeomorphic to $E_{+}^{3}$. By the above, $h(B)$ contains a neighborhood of $x$ in $L / G$ homeomorphic to $E_{+}^{3}$. Thus $L / G$ is a 3-manifold with boundary.

\section{REFERENCES}

1. P. Alexandroff, On local properties of closed sets, Ann. of Math. 36 (1935), 1-35.

2. R. H. Bing, The Klein sphere characterization problem, Bull. Amer. Math. Soc. 52 (1946), 644-653.

3. - Locally tame sets are tame, Ann. of Math. 59 (1954), 154-158.

4. — Approximating surfaces with polyhedral ones, Ann. of Math. 65 (1957), 456-483.

5. - - A surface is tame if its complement is 1-ULC, Trans. Amer. Math. Soc. 101 (1961), 294-305.

6. R. H. Bing and A. Kirkor, An arc is tame in 3-space if and only if it is strongly cellular, Fund. Math. 55 (1964), 175-180.

7. P. H. Doyle, On the embedding of complexes in 3-space, Illinois J. Math. 8 (1964), 615-620. 
8. E. Dyer and M. E. Hamstrom, Completely regular mappings, Fund. Math. 45 (1958), 103-108.

9. C. H. Edwards, Jr., Concentricity in 3-manifolds, Trans. Amer. Math. Soc. 113 (1964), 406-423.

10. H. C. Griffith and L. R. Howell, Jr., Strongly cellular cells in $E^{3}$ are tame (unpublished).

11. H. Kneser, Eine bermerkung uber dreidimensionalen mannigfaltigkeiten, Nachr. Ges. Wiss. Gottingen (1925), 128-130.

12. K. W. Kwun and F. Raymond, Mapping cylinder neighborhoods, Michigan Math. J. 10 (1963), 353-357.

13. Edwin E. Moise, Affine structures in 3-manifolds. VIII. Invariance of knot types; Local tame embedding, Ann. of Math. 59 (1954), 159-170.

14. R. L. Moore, Concerning upper semicontinuous collections of continua which do not separate a given set, Proc. Nat. Acad. Sci. U.S.A. 10 (1924), 356-360.

15. C. D. Papakyriakopoulos, On solid tori, Proc. London Math. Soc. (3) 7 (1957), 281-299.

16. _ Dehn's lemma and asphericity of knots, Ann. of Math. 66 (1957), 1-26.

17. F. Raymond, Separation and union theorems for generalized manifolds with boundary, Michigan Math. J. 7 (1960), 7-21.

18. W. Voxman, On the shrinkability of decompositions of 3-manifolds, Thesis, The University of Iowa, 1968.

19. R. L. Wilder, Topology of manifolds, Amer. Math. Soc. Colloq. Publ., Vol. 32, Amer. Math. Soc., Providence, R. I., 1963.

THE UNIVERSITY OF IOWA, IOWA CiTY, IOWA

Kent State UNiversity, KENT, OHIO 